\section{HIV Infection Presenting with Stroke and Epilepsia Partialis Continua}

Till date there are only few case reports of HIV infection in children, presenting with stroke and none with epilepsia partialis continua (EPC) as their first manifestation $[1,2]$. EPC as a neurological manifestation of HIV has been described previously, only rarely in children $[3,4]$. Here we report a child presenting with stroke and EPC, later diagnosed to be infected with HIV.

A 6-year old boy, developmentally normal, admitted with illness beginning with fever and abnormal jerky movements of right upper limb lasting initially for few seconds, progressing over next few days to involve right lower limb followed by involvement of right half of face. Seizures, at presentation, persisted during most times with amplitude decreasing during sleep. Child on examination had cervical lymph nodes palpable, liver 2 $\mathrm{cm}$ and spleen $2 \mathrm{~cm}$ palpable below costal margins. Patient had reduced level of alertness without meningeal signs and normal fundus. Child had right upper motor neuron facial nerve palsy, weak gag, aphasia and right hemiplegia. Reflexes were exaggerated in all four limbs with ankle clonus present. Lab investigation revealed prothrombin time 11 seconds, partial thromboplastin time 22 seconds, total serum protein $9.4 \mathrm{~g} \%$, albumin to globulin ratio 2.3/7.1, HIV ELISA positive, anti nuclear antibody negative, toxoplasma IgG $1: 1600$ and IgM $<1: 800$, VDRL non reactive, anticardiolipin antibodies and factor V Leiden mutation absent. Contrast MRI of the brain (34th day of illness) revealed infarct involving left basal ganglia and left insular region (middle cerebral artery territory) and frontal cortex (anterior cerebral artery territory) with MRA showing no evidence of any vascular malformation. Multivoxel spectroscopy was suggestive of bilateral basal ganglia infarct. Later patient's sib and both parents were tested and found positive for HIV. Cerebrospinal fluid (CSF) examination (1 month of illness) results showed leucocytes absent, proteins $38.2 \mathrm{mg} / \mathrm{dL}$, sugar $39 \mathrm{mg} / \mathrm{dL}$, Gram stain negative, culture sterile, cryptococcus (India ink staining), Japanese encephalitis (PCR and serology), Herpes simplex virus 1 and 2 (PCR \& serology), tuberculosis (adenosine deaminase, culture and PCR) were found to be negative. Electroencephalogram (EEG) was suggestive of diffuse encephalopathy left more than right with focal epileptiform discharges. Cardiac echocardiography was normal.

Cerebrovascular complications are associated with perinatal HIV infection, albeit as a rare presentation. Mechanisms underlying the increased risk for ischemic stroke in HIV infected include opportunistic infections, meningitis and vasculitis, primary HIV vasculopathy, altered coagulation, and cardioembolic events [5]. In absence of vasculitis, HIV-related vasculopathy may cause stroke, which was the most likely etiology in our case.

Ashwin Rajendra Saboo and Pratibha Singhi Department of Pediatrics, Post Graduate Institute of Medical Education and Research (PGIMER), Chandigarh, India.ashwinrsaboo@gmail.com

\section{REFERENCES}

1. Visudtibhan A, Visudhiphan P, Chiemchanya S. Stroke and seizures as the presenting signs of pediatric HIV infection. Pediatr Neurol. 1999;20:53-6.

2. Lodha R, Upadhyay A, Kapoor V, Kabra SK. Clinical profile and natural history of children with HIV infection. Indian J Pediatr. 2006;73:201-4.

3. Bartolomei F, Gavaret M, Dhiver C, Gastaut JA, Gambarelli D, Figarell-Branger D, et al. Isolated, chronic, epilepsia partialis continua in an HIV-infected patient. Arch Neurol. 1999;56:111-4.

4. Ferrari S, Monaco S, Morbin M, Zanusso G, Bertolasi L, Cerini R, et al. HIV-associated PML presenting as epilepsia partialis continua. J Neurol Sci. 1998;161:180-4.

5. Dobbs MR, Berger JR. Stroke in HIV infection and AIDS. Expert Rev Cardiovasc Ther. 2009;7:1263-71.

\section{Rickettsial Diseases}

We read with interest the article on Rickettsial diseases by Rathi, et al. [1]. The author mentions that no case of Ricketssia has been reported from Madhya Pradesh. We herein report a child with features suggestive of rickettsial infection, whom we recently managed.

A 1-year 6 month old child from Shajapur, MP, presented to us with a history of fever for 20 days. On day 3 , she had developed a generalized rash starting from the 